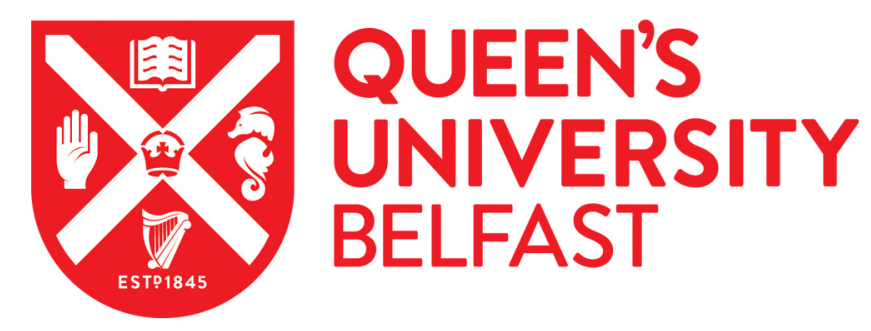

\title{
Ultra-High-Resolution Observations of MHD Waves in Photospheric Magnetic Structures
}

Jess, D. B., \& Verth, G. (2016). Ultra-High-Resolution Observations of MHD Waves in Photospheric Magnetic Structures. In A. Keiling, D-H. Lee, \& V. Nakariakov (Eds.), Low-Frequency Waves in Space Plasmas (pp. 449465). (Geophysical Monograph Series). John Wiley \& Sons Inc.. https://doi.org/10.1002/9781119055006.ch26

\section{Published in:}

Low-Frequency Waves in Space Plasmas

Document Version:

Publisher's PDF, also known as Version of record

Queen's University Belfast - Research Portal:

Link to publication record in Queen's University Belfast Research Portal

Publisher rights

Copyright @ 2016 American Geophysical Union.

\section{General rights}

Copyright for the publications made accessible via the Queen's University Belfast Research Portal is retained by the author(s) and / or other copyright owners and it is a condition of accessing these publications that users recognise and abide by the legal requirements associated with these rights.

Take down policy

The Research Portal is Queen's institutional repository that provides access to Queen's research output. Every effort has been made to ensure that content in the Research Portal does not infringe any person's rights, or applicable UK laws. If you discover content in the Research Portal that you believe breaches copyright or violates any law, please contact openaccess@qub.ac.uk. 


\title{
26 \\ Ultra-High-Resolution Observations of MHD Waves in Photospheric Magnetic Structures
}

\author{
D. B. Jess ${ }^{1}$ and G. Verth ${ }^{2}$
}

\subsection{INTRODUCTION}

Ever since oscillatory motion was discovered in the Sun's tenuous atmosphere [Leighton, 1960; Leighton et al., 1962; Noyes and Leighton, 1963], it has been a goal among physicists to detect, identify, characterize, and understand the diverse variety of wave modes manifesting in the solar atmosphere. Initial observations in the optical portion of the electromagnetic spectrum allowed oscillations present in the photospheric and chromospheric layers to be studied. However, during those early stages, technology was not as advanced as it is today, and as a result key modern techniques, such as adaptive optics (AO) [e.g., Rimmele and Marno, 2011], multi-object, multi-frame blind deconvolution (MOMFBD) [van Noort et al., 2005], and speckle reconstruction [Wöger et al., 2008], were unavailable to help combat the fine-scale image degradation caused by the Earth's atmosphere. Therefore the initial research was dedicated to probing large-scale solar structures, including sunspots and supergranules. However, even at these large spatial scales, a wealth of oscillatory phenomena was found to be omnipresent [e.g., Deubner and Liedler, 1969; Ulrich, 1970; Deubner, 1971]. Such waves demonstrated periodic intensity and velocity fluctuations, and thus were placed under the same umbrella as acoustic modes, which are ultimately defined by their intrinsic signatures of compressions and rarefactions. Difficulties were encountered in follow-up work when the measured phase velocities of the waves

${ }^{1}$ Astrophysics Research Centre, School of Mathematics and Physics, Queen's University Belfast, Belfast, Northern Ireland, UK

${ }^{2}$ Solar Physics and Space Plasma Research Centre $\left(S P^{2} R C\right)$, The University of Sheffield, Sheffield, UK were found to be too large to be explained by oversimplistic acoustic modeling. Instead, Osterbrock [1961] and Mein and Mein [1976] hypothesized that the magnetic fields in which the waves were embedded must also be taken into consideration.

Over the next decades, the examination of magnetoacoustic waves rose to the forefront of observational solar physics, with Ulmschneider [1976] aptly asking whether they may provide a significant channel for energy leaking into the outer layers of the Sun's atmosphere. Furthermore the mere presence of a magnetic field introduces a number of additional viable wave modes that may simultaneously exist within the atmosphere. Through magnetohydrodynamic (MHD) modeling it was found that although these additional waves have similarities with purely acoustic modes, they are often highly anisotropic. This is because the addition of the magnetic field introduces a dependency on both the alignment of the wavevector, $k$, with the direction of the background magnetic field, $B_{0}$, and the ratio of the kinetic pressure, $p_{0}$, to the magnetic pressure, $B_{0}^{2} / 2 \mu_{0}$ (or $B_{0}^{2} / 8 \pi$ in cgs units), in the environment that supports the wave. This ratio is commonly referred to as the plasma $\beta$, defined as $\beta=2 \mu_{0} p_{0} / B_{0}^{2}$, where $\mu_{0}$ is the magnetic permeability of free space. This quantity can be rewritten in terms of the local hydrogen number density, $n_{H}$, the plasma temperature, $T$, and the Boltzmann constant, $k_{B}$, as $\beta=8 \pi n_{H} T k_{B} / B_{0}^{2}$, providing a representation of the plasma $\beta$ in cgs units. Thus in the lower layers of the solar atmosphere where the temperature is relatively low $(T \sim 6000 \mathrm{~K})$ and the magnetic field strength is still intensely concentrated $\left(B_{0}>1000 \mathrm{G}\right)$, a majority of the magnetic structures supporting magnetohydrodynamic wave phenomena are often defined by $\beta \ll 1$ (i.e., dominated by magnetic pressure). This has

Low-Frequency Waves in Space Plasmas, Geophysical Monograph 216, First Edition.

Edited by Andreas Keiling, Dong-Hun Lee, and Valery Nakariakov.

(C) 2016 American Geophysical Union. Published 2016 by John Wiley \& Sons, Inc. 
important consequences for the wave modes that are expected to exist within this plasma regime, and allows for the manifestation of "fast" and "slow" magnetoacoustic waves, in addition to Alfvén waves [Edwin and Roberts, 1983]. Structures in the lower solar atmosphere that demonstrate oscillatory behavior are often observed to be elongated (mottles, spicules, fibrils, etc.), and as such are typically modeled by employing cylindrical geometry. Here an overdense magnetic "flux tube" will create an efficient waveguide that will support an even richer variety of MHD waves depending on the azimuthal wavenumber, $m$, including sausage $(m=0)$, kink $(m=1)$ and fluting modes $(m>1)$, which are yet further classified via their trapped/leaky, fast/slow and body/surface characteristics. Thus the quest to detect all of the various MHD wave modes has intensified, but so too has the drive to determine the precise role they play in the transportation and dissipation of energy through the Sun's atmosphere.

The ongoing research into waves and oscillations in the solar atmosphere does not take place for solely esoteric purposes. Rather, it has enabled us to delve deep below the visible solar surface through helioseismology techniques [e.g., Duvall et al., 1993, Schou et al., 1998; Lopes and Silk, 2014], and to understand the coupling between (quasi-) periodic flows and plasma motions that are abundantly apparent over a wide range of spatial scales (spicules, mottles, fibrils, plumes, prominences, etc.). When quantified, the possible contribution of observed MHD waves to plasma heating is also of immense interest in solar physics. The paradoxical nature of how the Sun's outer atmosphere is heated to (and maintained at) multi-million degree temperatures is a problem that has been plaguing scientists for over half a century. Even the solar chromosphere, a thin atmospheric layer that is only heated to a few thousand degrees above the underlying photosphere, requires extraordinary plasma heating processes to balance the radiative losses experienced in this relatively high-density environment. It has long been believed that MHD waves, generated in the photosphere and channeled upward along magnetic field lines, may be able to contribute directly to plasma heating providing a suitable (and efficient) conversion mechanism exists.

To generate localized heating from MHD waves, smaller length scales must be created via physical processes such as resonant absorption, phase mixing, or shock formation. Observing such fine-scale plasma dynamics directly presents a significant observational challenge due to the near- (or sub-) resolution scales involved. De Pontieu et al. [2004] demonstrated how ubiquitous $p$-mode oscillations can be channeled along magnetic field lines, ultimately giving rise to dynamic phenomena in the chromosphere and transition region. Furthermore Hansteen et al. [2006] revealed how shocks can form when slow magnetoacoustic waves, which are generated by convective flows and global $p$-mode oscillations, leak upward from the solar surface along magnetic field lines and encounter the steep density gradients intrinsic to the chromosphere. Of significant importance is the fact that there is now overwhelming evidence to suggest that waves have the ability to deform magnetic field lines and induce the necessary instabilities required to incite reconnective phenomena over a wide range of atmospheric heights [e.g., Isobe and Tripathi, 2006; Isobe et al., 2007; Jess et al., 2010a; Li and Zhang, 2012; Jackiewicz and Balasubramaniam, 2013; Shen et al., 2014]. Thus examining the generation and behavior of oscillatory phenomena in the solar atmosphere has the potential to shine light on a wide variety of physical phenomena, from magnetic reconnection on sub-arcsecond scales through to prominence eruptions spanning many hundreds of arcseconds.

In more modern times, the advent of high-sensitivity and low-noise camera systems has allowed short-exposure and high-cadence image sequences to be obtained from both ground- and space-based observatories. In particular, for ground-based solar telescopes such as the Dunn Solar Telescope (DST) and the Swedish Solar Telescope (SST), the successful application of adaptive optics and post-processing techniques has allowed researchers to focus their attention on the smallest magnetic elements observed in the Sun's lower atmosphere. Features that are close to the resolution limits of current solar telescopes, including magnetic bright points (MBPs) [Dunn and Zirker, 1973; Stenflo, 1985; Solanki, 1993], offer unique advantages over more large-scale structures (e.g., sunspots) in the study of MHD wave phenomena. For instance, these omnipresent elements, easily identifiable and ideal for feature-tracking algorithms, are not as magnetically complex as macro-sized structures such as sunspots, and are therefore more readily compared with cylindrical geometry MHD approximations. Furthermore, due to their small size (often less than $0.3^{\prime \prime}$ or $220 \mathrm{~km}$ in diameter; Crockett et al. [2010]), these structures are more prone to the buffeting imposed by the complex evolution of surrounding granules, thus increasing the likelihood of generating wave motion at the photospheric base of the magnetic field lines. In addition the magnetic field strengths associated with MBP structures are of comparable magnitude to those found in large-scale sunspots $(\geq 1 \mathrm{kG}$; Cauzzi et al., 2000; Jess et al., 2010b), making them extremely viable conduits for carrying a variety of MHD waves, including fast and slow magnetoacoustic modes as well as Alfvén waves. MBPs have demonstrated their ubiquity in both observational and simulated datasets, with an example of such features displayed in Figure 26.1.

The simultaneous development of theoretical, numerical, and analytical modeling tools for the lower solar 


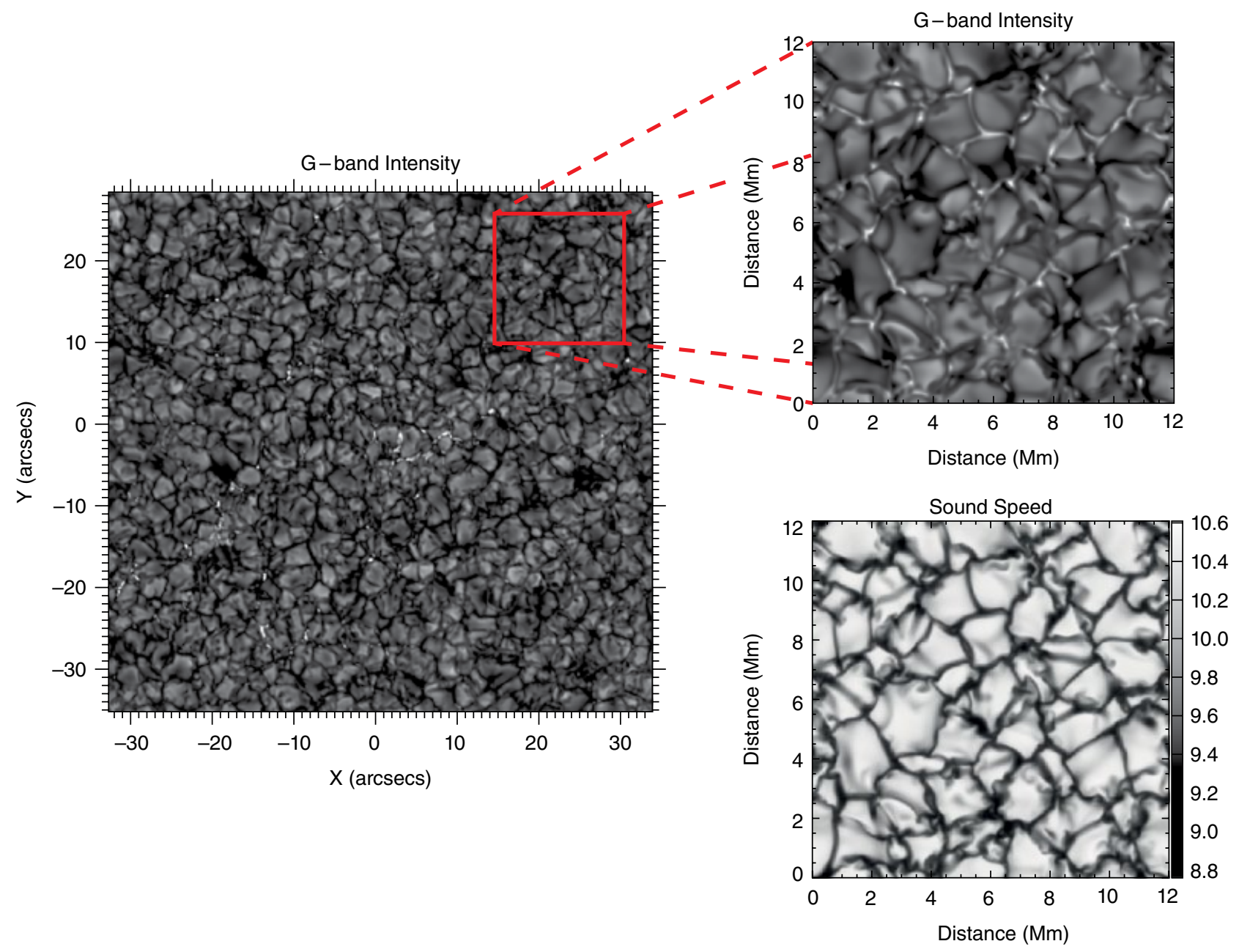

Figure 26.1 G-band image, acquired by the ROSA multi-camera system at the Dunn Solar Telescope, revealing a large number of MBPs. These features are visible as intensity enhancements within the intergranular lanes, and often demonstrate magnetic field strengths exceeding $1000 \mathrm{G}$. The axes are in heliocentric arcseconds, where $1^{\prime \prime} \sim 0.725 \mathrm{Mm}$. The red square highlights a $12 \times 12 \mathrm{Mm}^{2}$ subregion of the field of view, with an equally sized simulated G-band image. The output of the MuRAM numerical code also reveals bright magnetic features manifesting within the intergranular lanes, suggesting a significant amount of agreement between current high-resolution observations and radiative magnetohydrodynamic modeling techniques. Local sound speeds calculated from the local densities and pressures in the simulated polytropic atmosphere. It is clear that intergranular lanes, where magnetic bright points reside, often display sound speeds on the order of $10 \mathrm{kms}^{-1}$. Images adapted from Jess et al. [2012c].

atmosphere has allowed the unification of MHD with seismological techniques [e.g., Kim et al., 2008; Verth et al., 2011; Kuridze et al., 2013; Morton et al., 2014]. Observers are now able to quantify and understand oscillatory parameters that may be below the resolution limit imposed by even the largest of modern ground-based optical telescopes (e.g., the $1.6 \mathrm{~m}$ New Solar Telescope, NST, at the Big Bear Solar Observatory). This has important consequences, particularly when attempting to identify multiple modes existing within the same structure [e.g., Morton et al., 2012], or when trying to diagnose small-amplitude waves that may often become swamped by instrumental noise and/or the point spread function of the telescope. In this chapter, we will review the recent observations of waves and oscillations manifesting in fine-scale magnetic structures in the solar photosphere, which are often interpreted as the "building blocks" of the magnetic Sun.

\subsection{MAGNETOACOUSTIC WAVES}

The launch of the Hinode [Kosugi et al., 2007] space telescope, equipped with the $0.5 \mathrm{~m}$ Solar Optical Telescope (SOT) [Suematsu et al., 2008; Tsuneta et al., 
2008], provided researchers with easily accessible and high-resolution $\left(\sim 0.2^{\prime \prime}\right.$ or $\left.150 \mathrm{~km}\right)$ observations of the lower solar atmosphere. The lack of atmospheric turbulence results in the images being "seeing free," and thus suitable for long duration studies of small-scale magnetic elements on the surface of the Sun. Notably, Carlsson et al. [2007] were at the forefront of employing the highresolution $\mathrm{Ca}$ II $\mathrm{H}$ and continuum filters onboard SOT to examine the propagation of acoustic waves down to the diffraction limit of the instrumentation. Through examination of all spatial locations (both magnetic and nonmagnetic), Carlsson et al. [2007] speculated that the total energy flux provided, even at such high spatial and frequency resolutions, was insufficient to contribute to atmospheric heating. However, Wedemeyer-Böhm et al. [2007] stated that the methods used may overlook dynamic patterns created on subresolution scales, and as a result severely underestimate the actual mechanical flux [Kalkofen, 2007, 2008]. Importantly, this work inspired many other solar scientists to focus on highly magnetic photospheric elements, where the strong magnetic flux concentrations may promote more efficient energy propagation.

Employing high spatial and temporal resolution observations from the ground-based DST, Jess et al. [2007] detected more prevalent photospheric oscillations in what was believed to be magnetic concentrations surrounding a large sunspot. These oscillations demonstrated power well in excess of the quiescent background, but unfortunately, no direct information on the associated magnetic fields were available, and thus the waves were tentatively tied to magnetoacoustic phenomena. Further work by Andic [2007] found that the locations of small-scale oscillatory power in the photosphere correlated well with red-shifted velocities (i.e., downflows). This is in agreement with the theory that many small-scale magnetic elements in the photosphere are formed via the process of convective collapse [Spruit, 1976], which is further substantiated by the modern-day observations and MHD simulations of MBPs [e.g., Utz et al., 2013; Hewitt et al., 2014]. However, it must be stated that while such oscillations have been tied to MBPs displaying red-shifted (i.e., downflow) velocities, this does not necessarily mean that the embedded waves are also downwardly propagating. A bulk motion may exist within the confines of the magnetic flux tube, hence giving rise to red-shifted Doppler signatures, yet the phase velocity of the wave may have a sufficiently large upward magnitude such that the overall group velocity, which crucially describes the velocity at which energy is propagated by the wave, is directed upward. For example, the observational work of Andić [2007] and Narayan [2011], corroborated by the numerical modeling presented by Hewitt et al. [2014], established downflow velocities on the order of a few $\mathrm{kms}^{-1}$ within the confines of small-scale photospheric magnetic elements (e.g., see the upper panels of Figure 26.2). These subsonic plasma flows may easily become overshadowed by upwardly propagating magnetoacoustic phase velocities that are close to the photospheric sound speed $\left(\sim 10 \mathrm{kms}^{-1}\right.$; Jess et al. [2012c]). As a result the group velocity, and therefore the direction of energy propagation, would be directed upward. Recently Kato et al. [2011] further revealed how the processes intrinsic to convective collapse may also drive magnetoacoustic wave phenomena in small-scale magnetic elements, regardless of the presence of subsurface $p$-mode oscillations. The authors employed radiative MHD simulations and demonstrated how the coupling between external downdrafts in the intergranular lanes and the motions of the embedded plasma relies heavily on the inertial forces that act on the magnetic flux concentration. These forces act to "pump" the internal atmosphere of the magnetic flux tube in a downward direction, which eventually causes the atmosphere to rebound, producing upwardly propagating magnetoacoustic waves along the magnetic field lines [Kato et al., 2011].

Through examination of the magnetoacoustic wave dynamics associated with large-scale sunspots, Nagashima et al. [2007] employed Hinode/SOT observations to reveal how oscillatory power is often drastically reduced in the presence of strong magnetic field concentrations; a common phenomenon now referred to as "acoustic power suppression" [e.g., Woods and Cram, 1981; Thomas et al., 1982; Title et al., 1992; Parchevsky and Kosovichev, 2007; Chou et al., 2009; Ilonidis and Zhao, 2011; Couvidat, 2013]. Lawrence and Cadavid [2010, 2012] and Chitta et al. [2012a] were able to corroborate these general findings through examination of Hinode/SOT G-band image sequences containing MBPs. However, interestingly Chitta et al. [2012a] found evidence to suggest that magnetoacoustic power at the highest temporal frequencies (i.e., periodicities less than $100 \mathrm{~s}$ ) actually demonstrated power amplification. Follow-up work, employing the Rapid Oscillations in the Solar Atmosphere (ROSA) [Jess et al., 2010c] multi-camera imaging system on the DST, revealed how magnetoacoustic waves with periodicities below $100 \mathrm{~s}$ demonstrate significant turbulent components within their power spectra [Lawrence et al., 2011]. Lawrence et al. [2011] suggest that the observed magnetoacoustic waves may be generated by the interaction of the magnetic field lines with plasma downflows (i.e., characteristic of the convective collapse process) that are very turbulent in their nature.

While the observed periodicities of magnetoacoustic waves in MBPs generally span the entire $p$-mode spectrum, there is increasing evidence to suggest that the underlying magnitude of the magnetic field directly influences the dominant period. Kostik and Khomenko [2013] employed 



Figure 26.2 Simultaneous G-band intensity (upper left) and line-of-sight velocity (upper right) sub-fields extracted from a synthesized field of view created using the MuRAM radiative magnetohydrodynamic code. Each image covers less than 1 square arcsecond, and is centered on a recently formed MBP structure manifesting within the intergranular lanes of the surrounding convective plasma. The red contours in the upper left panel outline locations where the downflow (i.e., red-shifted) velocities exceed $3 \mathrm{kms}^{-1}$, as defined by the graduated color spectrum displayed at the right-hand side of the figure. The red-shifted plasma encompasses both the MBP and the surrounding intergranular lanes, indicating the process of convective collapse is likely to play an important role in the formation of MBP features. Note that the downflow velocities at the center of the MBP, where wave phenomena is likely to manifest as a result of the increased magnetic field strengths, are slightly weaker than at its perimeter. The lower panels display the occurrence of oscillations simultaneously visible in ROSA G-band and $4170 \AA$ continuum images, as a function of the oscillation period and phase angle for regions containing MBPs (lower left) and those without (lower right). The color scale represents the number of detections as a percentage of the total events with an associated coherence level exceeding 85\%. A horizontal dashed line represents a phase angle of 0 degrees, while dotted lines highlight a region inside which detections become unreliable due to cadence restrictions $(0.528 \mathrm{~s}$ for the observational time series). A preference for negative phase shifts highlights the abundance of upwardly propagating wave motion in nonmagnetic and magnetic photospheric features, including the small-scale MBPs shown in the upper panels. Upper images courtesy of R. L. Hewitt, and based on the data presented by Hewitt et al. [2014]; the lower panels adapted from Jess et al. [2012c].

the Triple Etalon SOlar Spectrometer (TESOS) [Trischler et al., 2002] on the German Vacuum Tower Telescope (VTT) to obtain high-resolution spectroscopy of the photospheric Ba II absorption line. They found that the dominant period of oscillations increases by $15-20 \%$ as the local magnetic field strength increases from 500 to $1500 \mathrm{G}$. This has important implications because it suggests that the strong magnetic fluxes inherent to MBPs may be able to assist the propagation of lower frequency (i.e., below $3 \mathrm{mHz}$ ) magnetoacoustic waves into the 
chromosphere and beyond, especially if the magnetic field lines are suitably inclined to reduce the impact of the acoustic cutoff frequency [i.e., Bel and Leroy, 1977]. As suggested by De Pontieu et al. [2004], de Wijn et al. [2009], and Stangalini et al. [2011], to name but a few, the efficient leakage of lower frequency magnetoacoustic wave modes into the upper solar atmosphere may be able to drive a wide variety of high-temperature phenomena, including the oscillations observed in coronal fans, plumes, and loops [e.g., Deforest and Gurman, 1998; Ofman et al., 1999; Ofman and Wang, 2002; De Moortel and Hood, 2003, 2004].

High-resolution observations have clearly shown the existence of magnetoacoustic wave phenomena in smallscale photospheric magnetic elements. However, it is also important to determine whether these oscillations are propagating or standing waves from the viewpoint of supplying energy to the upper levels of the solar atmosphere. Jess et al. [2012c] employed the ROSA system with blue continuum (4170 $)$ ) and G-band filters to examine the propagation of waves between two discreet layers in the lower solar atmosphere. The MuRAM radiative magnetohydrodynamic code [Vögler et al., 2005] was utilized to determine the formation heights of the two ROSA filtergrams through a comparison of their corresponding response functions. It was found that the continuum and G-band images were separated by $\sim 75 \mathrm{~km}$ in height, thus allowing any propagation of waves between these two discreet layers to be investigated through phase-difference analysis. Jess et al. [2012c] were able to detect a wealth of oscillatory phenomena in both continuum and G-band images. However, only oscillations with periodicities above $\sim 140$ s demonstrated coherent phase delays between the adjacent bandpasses. $\mathrm{A}-8^{\circ}$ phase lag (traversing a physical displacement of $\sim 75 \mathrm{~km}$ ) indicated upwardly propagating phase speeds on the order of $8 \mathrm{~km} \mathrm{~s}^{-1}$. These velocities are similar to the expected sound speed (Figure 26.1c), and thus demonstrated the linear nature of magnetoacoustic wave phenomena in the lower solar atmosphere. Furthermore, as revealed in Figures 26.2c and 26.2d, the authors determined that $76 \%$ of all MBP structures demonstrated upwardly propagating magnetoacoustic wave signatures, helping explain why the outer regions of the solar atmosphere are so ubiquitously populated with MHD wave phenomena. Indeed extensive work has recently been implemented to uncover the connectivity between lower atmospheric propagating waves and running oscillations ubiquitously observed in the solar corona [e.g., Tomczyk and McIntosh, 2009; Jess et al., 2012a].

More recently Andić et al. [2013] employed broadband TiO images obtained using the NST to examine the connection between photospheric oscillations and the dynamic motions of small-scale magnetic flux concentrations. The TiO filter used is centered on an absorption band of molecules around $7056.8 \AA$ (incorporating a 10 $\AA$ Ailter width), and thus averages over all inherent absorption and continua contributions, causing the resulting images to be only weakly dependent on the properties of individual spectral lines. Ultimately, this means that the intensity time series will be representative of the true solar continuum. Furthermore, since the bandpass is approaching the near-infrared, the images will be less sensitive to atmospheric seeing variability, and thus provide better temporal coverage of time series obtained during mediocre weather conditions. The authors undertook Fourier and Hilbert transformations of the TiO time series related to MBPs, in order to extract the amplitude and phase relationships of the embedded oscillations, and suggested that the detected wave motion is likely to be too complex to be generated by a single oscillatory source. Nevertheless, Andić et al. [2013] provided direct evidence for the presence of upwardly propagating wave trains in the immediate vicinity of red-shifted (i.e., downflowing) material, suggesting the phase velocities of the magnetoacoustic wave phenomena were significantly higher than $5 \mathrm{kms}^{-1}$ at the photospheric layer.

\subsubsection{Sausage Waves}

Even though the sausage mode is the lowest azimuthal order compressible mode (i.e., $m=0$ ), it has still proved extremely difficult to identify in observations. Through mathematical understanding and numerical modeling, these waves will demonstrate observational characteristics consistent with the simultaneous periodic intensity and area fluctuations of the magnetic flux tube. One of the main obstacles, at least observationally when attempting to detect sausage-mode oscillations, is a combination of the instrumental spatial resolution (i.e., to be able to detect the fractional area changes) in addition to the detector sensitivity (i.e., to be able to extract the smallscale intensity fluctuations over the intrinsic background noise). Only with the consistently high resolving power of modern solar facilities, coupled with the low-noise characteristics synonymous with cooled CCD and CMOS detectors, has it been possible to detect sausage waves in small-scale photospheric magnetic elements.

Fujimura and Tsuneta [2009] revealed the true power of the SOT onboard the Hinode spacecraft by examining the intensity and velocity oscillation characteristics in relation to the vector magnetic field (e.g., see Figures $26.5 \mathrm{~d}-26.5 \mathrm{f}$ ). The authors found, through phase relationships between the various waveforms, that smallscale MBPs in the photosphere demonstrated signatures of specific magnetoacoustic waves, in particular the sausage and kink modes. The observed fluctuations in the magnetic field, the line-of-sight velocity and the structure's intensity indicated root-mean-square amplitudes of 
$4-17 \mathrm{G}(0.3 \%-1.2 \%), 0.03-0.12 \mathrm{kms}^{-1}$, and $0.1 \%-1 \%$, respectively. The small amplitudes of the observed fluctuations emphasised the importance of high-resolution (spatial, temporal, and spectral) observations when attempting to diagnose certain magnetoacoustic wave modes. Importantly, the detected oscillations maintained significant overlap with the global $p$-mode periodicities, further confirming the assumption that the majority of wave motion found in the solar atmosphere are driven by the underlying and omnipresent $p$-mode oscillations.

Employing the spectropolarimetric capabilities of the Imaging Magnetograph eXperiment (IMaX) [Martínez Pillet et al., 2011] oboard the Sunrise balloon flight, Martínez González et al. [2011] established clear evidence for periodic fluctuations in the magnetic field strength associated with small-scale $\left(\leq 1^{\prime \prime}\right)$ elements within the intergranular lanes. The isocontours related to specific magnetic fluxes were observed to oscillate in both area and magnitude, with some antiphase behaviours suggesting the presence of sausage-mode waves [Fujimura and Tsuneta, 2009]. However, the authors found that the periodicities of the oscillations were seldomly constant, and in fact often varied by several minutes within the same location (Figure 26.3). As a result Martínez González et al. [2011] proposed that the wave driver may not be the expected $p$-mode oscillations, and instead may be a consequence of granular "forcing." It was proposed that since the average magnetic fields contained within the intergranular lanes might have strengths lower than the photospheric equipartition field ( 300 - 500G; Lin [1995];
Khomenko et al. [2003]; Martínez González et al. [2008]), the continuously buffeting nature of granular flows may directly impose periodic field amplification and weakening through the processes associated with plasma forcing. Importantly, as raised by Martínez González et al. [2011], is the question as to whether or not these fluctuations (regardless of whether they are driven by underlying $p$-modes or by granular forcing) are able to propagate upward through the solar atmosphere, thus facilitating the relocation of energy to higher atmospheric heights. Utilizing other magnetically sensitive absorption lines, particularly those originating in the chromosphere (e.g., the $\mathrm{Ca}$ II infrared triplet at $8542 \AA$ ), will allow such magnetic fluctuations to be tracked through the solar atmosphere and shine new light on whether they can act as an efficient energy conduit.

Inspired by the results of Fujimura and Tsuneta [2009], Moreels and Van Doorsselaere [2013a] and Moreels et al. [2013b] developed stringent phase relationships that allow the characterization of fast/slow, body/surface, and standing/propagating sausage-mode waves based on the measured delays between the intensity, cross-sectional area and velocity components of the plasma. These relationships were successfully applied to the observational work of Morton et al. [2011], which demonstrated their accuracy and suitability for interpreting particular sausagemode properties, such as standing/propagating, fast/ slow, and body/surface. However, the work of Morton et al. [2011] examined solar pores, which have diameters $>1250 \mathrm{~km}$ that are substantially larger than the $\sim 220 \mathrm{~km}$
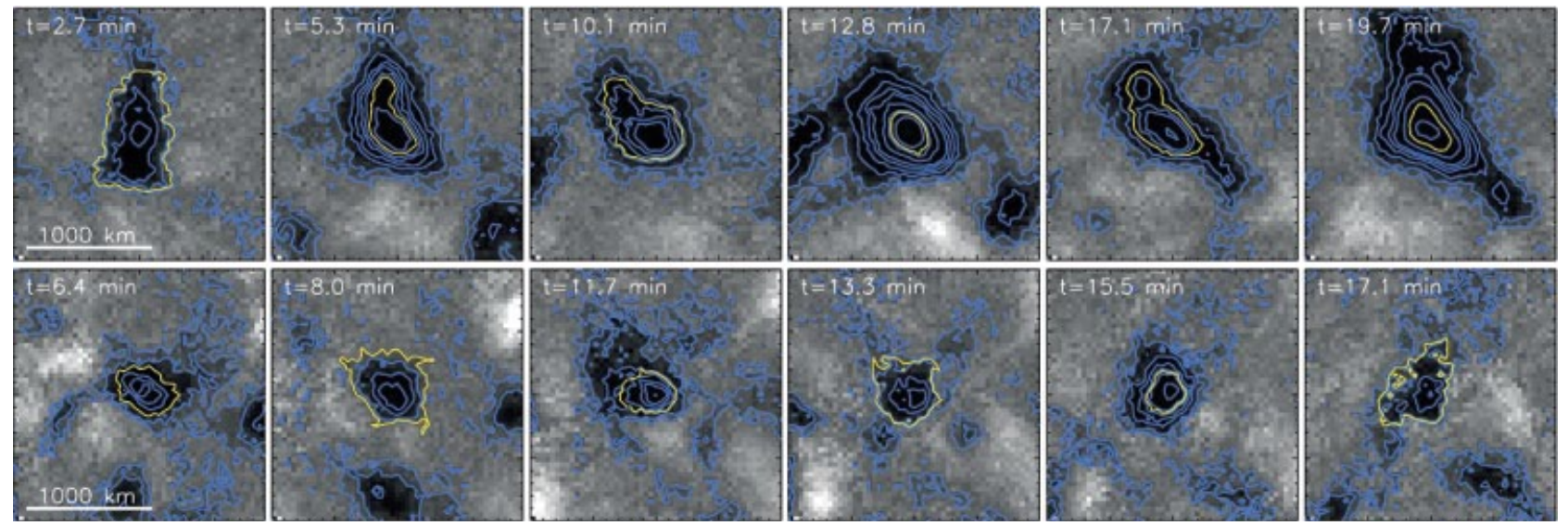

Figure 26.3 Panels displaying the time evolution of circularly polarized (i.e., longitudinal) magnetic fields in the photosphere, as captured by the IMaX instrument on-board the Sunrise balloon flight. What may initially appear to be pore-sized structures are in fact sub-arcsecond magnetic concentrations contained within the intergranular lanes, with white and black colors representing positive and negative magnetic flux densities, respectively, computed using the weak-field approximation. The blue contours highlight various iso-magnetic flux densities, and the yellow contours represent time-constant magnetic fluxes equal to $-4.5 \times 10^{16} \mathrm{Mx}$ (upper row) and $-5.0 \times 10^{16}$ Mx (lower row). It is clear that the time-constant iso-magnetic contours contract and expand with time, suggesting the presence of compressive magnetoacoustic waves embedded within the field lines. Images adapted from Martínez González et al. [2011]. 
sizes associated with the smallest scale magnetic elements in the photosphere [Crockett et al., 2010]. Consequently efforts are now being directed toward high-resolution observations of MBPs, which will allow the phase relationships to be tested more thoroughly for the smallest magnetic structures currently resolvable.

Jess et al. [2012b] examined the mode-coupling between compressible and incompressible waves found in MBPs, and the connection with their chromospheric spicle counterparts. The primary aim of this work was not to study sausage-mode oscillations, but instead their source. Using the Lare2D numerical code [Arber et al., 2001] to model an MBP as a thin magnetic flux tube, the authors found that a $90^{\circ}$ out-of-phase behavior of upwardly propagating magnetoacoustic waves at the photospheric layer directly incited the generation of sausage-mode oscillations in the same flux tube. Jess et al. [2012b] interpreted the numerical output as evidence for how velocity gradients embedded within the flux tube, as a result of the out-of-phase magnetoacoustic oscillations, cause the central axis of the magnetic fields to displace transversally. In addition, compressions and expansions in the waveguide are simultaneously induced, thus promoting the manifestation of both compressible sausage modes and incompressible kink waves at upper photospheric heights. The work of Jess et al. [2012b] clearly shows how thin, magnetic structures omnipresent throughout the solar atmosphere can readily support sausage-mode wave generation and propagation, which is in agreement with the chromospheric work of Morton et al. [2012].

\subsubsection{Kink Waves}

The $m=1$ kink mode is unique in that it is the only value of the azimuthal wavenumber, $m$, that produces a transverse displacement of a magnetic flux tube. Hence it is readily observed in overdense solar structures with imagers of sufficient spatial and/or temporal resolution. The kink mode is highly Alfvénic, since its main restoring force is magnetic tension, thereby making it only weakly compressible [e.g., see Goossens et al., 2009, for a detailed discussion]. Kink waves have been most extensively studied in the corona following the launch of the Transition Region and Coronal Explorer (TRACE) [Handy et al., 1999] spacecraft in 1998. Since 2007 they have also become the subject of much interest in the chromosphere due to their heightened visibility in off-limb spicules with Hinode. Since propagating kink waves in both chromospheric and coronal waveguides are now seen to be ubiquitous, it is widely believed they are being driven from the wealth of mechanical energy permeating the photosphere. As a result kink waves have become hypothesized as a favorable transport mechanism for channeling energy from the photospheric convective motions through to the upper layers of the solar atmosphere [e.g., Cranmer and van Ballegooijen, 2005; Suzuki and Inutsuka, 2005; Verdini and Velli, 2007; Matsumoto and Shibata, 2010]. Investigations into their possible photospheric signatures are now gaining momentum. There is some tentative observational evidence of the kink instability in penumbral filaments surrounding sunspots [e.g., Ryutova et al., 2008; Bharti et al., 2012], but thus far there has been no conclusive statistical studies undertaken of kink waves propagating along such structures. However, since penumbral filaments are predominantly highly inclined and/or closed magnetic structures confined to the lower atmosphere, they do not represent the conduits required to transfer kink wave energy into the corona. A much more promising form of investigation is to track the photospheric motions that could instigate kink waves. Since fine-scale chromospheric magnetic structures, such as spicules, fibrils, and mottles, are rooted in intergranular lanes, their foot points often reveal themselves in photospheric G-band filtergrams as MBPs [Jess et al., 2012b]. Tracking the horizontal velocity components of MBPs provides a useful proxy for detecting the transverse wave drivers that can excite kink modes, assuming that the inclinations of the associated magnetic flux tubes are not too far from the vertical at photospheric heights. The advent of high temporal and spatial resolutions from modern facilities has allowed observers to measure such small-scale transverse exertions, and as a result the quest is now on to relate such photospheric horizontal velocity power spectra with that of kink oscillations observed higher up in the chromosphere and corona. A key scientific goal is now to provide an all-encompassing understanding of kink wave excitation, propagation and damping throughout the entire solar atmosphere.

In a series of recent observational papers, Stangalini et al. [2013a, b, 2014] employed data, acquired by the Hinode/SOT and the Sunrise/IMaX instruments, to examine the interactions between MBPs and their surrounding granular environment in an attempt to uncover how transverse waves in the photosphere are generated. First, Stangalini et al. [2013a] found an abundance of photospheric magnetic elements demonstrating buffetinginduced transverse oscillations with periodicities under $100 \mathrm{~s}$ and velocity amplitudes of the order of $1-2 \mathrm{kms}^{-1}$. The authors interpreted their results as evidence for kink waves being generated by granular buffeting, and accompanied by longitudinal magnetoacoustic oscillations generated via nonlinear interactions. The results put forward by these authors corroborate numerous theoretical and numerical studies that suggest such motions to be a natural response of the magnetic field to external plasma forcing [e.g., Roberts, 1983; Steiner et al., 1998; Hasan et al., 2003; Musielak and Ulmscheider, 2003; Khomenko et al., 2008; Fedun et al., 2011a; Morton et al., 2014]. 
(a)

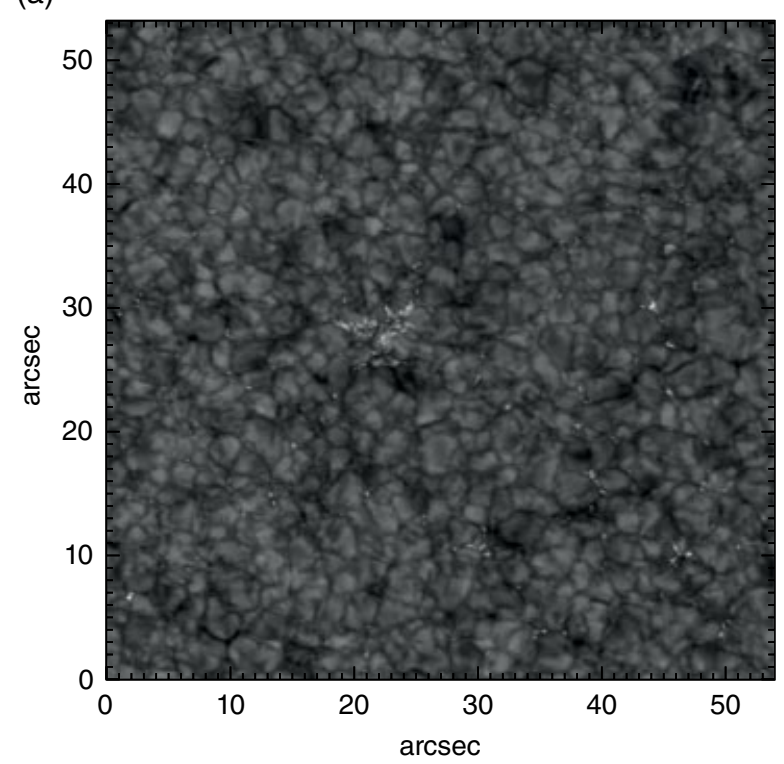

(b)

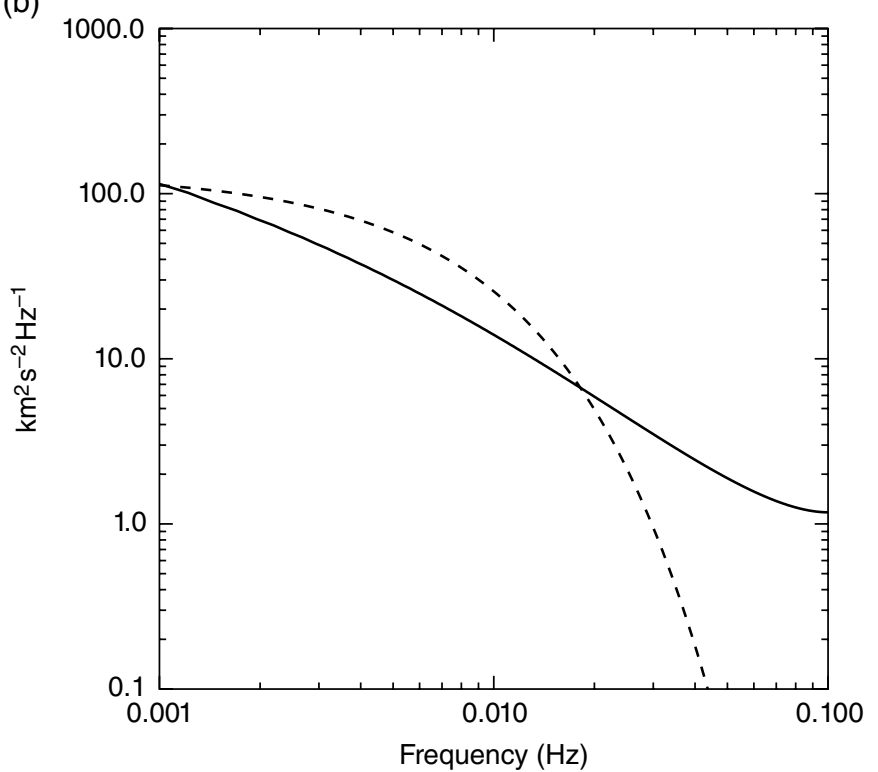

Figure 26.4 (a) Broadband $\mathrm{H} \alpha$ image acquired by the SST on 18 June 2006, revealing a large assortment of MBPs within the intergranular lanes. (b) Following correlation tracking on each of the 97 detected MBPs within the field of view, the resulting power spectrum of the horizontal motions is displayed as a function of frequency (solid black line). The dashed line represents a standardized Lorentz profile using identical free parameters (e.g., the correlation time) to that measured in the SST observations. It is clear that for frequencies $>0.02 \mathrm{~Hz}(<50 \mathrm{~s})$ the observational horizontal motions have more power, highlighting the fact that dynamics on short timescales may be very important in the generation of energetic kink and/or Alfvén wave phenomena. Images adapted from Chitta et al. [2012b].

Of particular note is the related observational work of Keys et al. [2011], who found that at any one time, approximately $6 \%$ of photospheric MBPs display transversal velocities exceeding $2 \mathrm{kms}^{-1}$, which according to the mathematical analyses of Choudhuri et al. [1993a, b], is more than sufficient to effectively and efficiently drive kink-mode oscillations in photospheric magnetic elements.

Chitta et al. [2012b] employed broadband $\mathrm{H} \alpha$ images, acquired by the SST with a cadence of $5 \mathrm{~s}$, to calculate the flow velocities of MBPs, relative to their local environment, through correlation tracking algorithms. The broadband nature of the $\mathrm{H} \alpha$ filter ( $8 \AA$ full-width halfmaximum) resulted in the images being dominated by photospheric contributions, as can be seen in Figure 26.4a. The authors were able to detect, track and analyze 97 individual MBP features, which allowed them to calculate the power spectrum of horizontal (i.e., transverse) fluctuations at the photospheric level. When the observational power spectrum was compared to a standardised Lorentzian model (e.g., see Figure 26.4b), it was found that the Lorentzian power spectrum grossly underestimated the power originating within oscillations $>0.02 \mathrm{~Hz}$ (i.e., $<50 \mathrm{~s}$ periodicity). This work suggests that MBP dynamics on short timescales may be very important in the generation of highly energetic kink and/or Alfvén wave phenomena. Furthermore, utilizing both ground- and space-based observatories, Morton et al. [2013] presented observations that revealed how kink waves can also be excited by the vortex motions of a strong magnetic flux concentration in the solar photosphere (Figure 26.5). The authors detected considerable horizontal flows, in addition to evidence for torsional vorticities, in the highresolution observational ROSA data and suggested that these may instigate considerable wave motion in the lower atmosphere and beyond. To test their hypothesis, Morton et al. [2013] computed photospheric flow vectors from complementary MuRAM simulations, which also indicated that small vortical movements of the photospheric plasma, with magnitudes up to $\sim 0.3^{\circ} \mathrm{s}^{-1}$, can help generate the kink waves observed in both high-resolution simulations and observations. With MBPs covering an estimated 2.2\% of quiet Sun locations [Sánchez Almeida et al., 2010], it does not seem inconceivable that the generation of kink motions in such small-scale magnetic fields may be responsible for the delivery of significant energy to higher atmospheric layers.

Taking this one step further, and employing the long-duration observations provided by the spaceborne Hinode/SOT instrument, Stangalini et al. [2013b] examined the spectral characteristics (in the Fourier domain) of small-scale magnetic elements undergoing kink-like oscillations. Interestingly, the authors found that while 

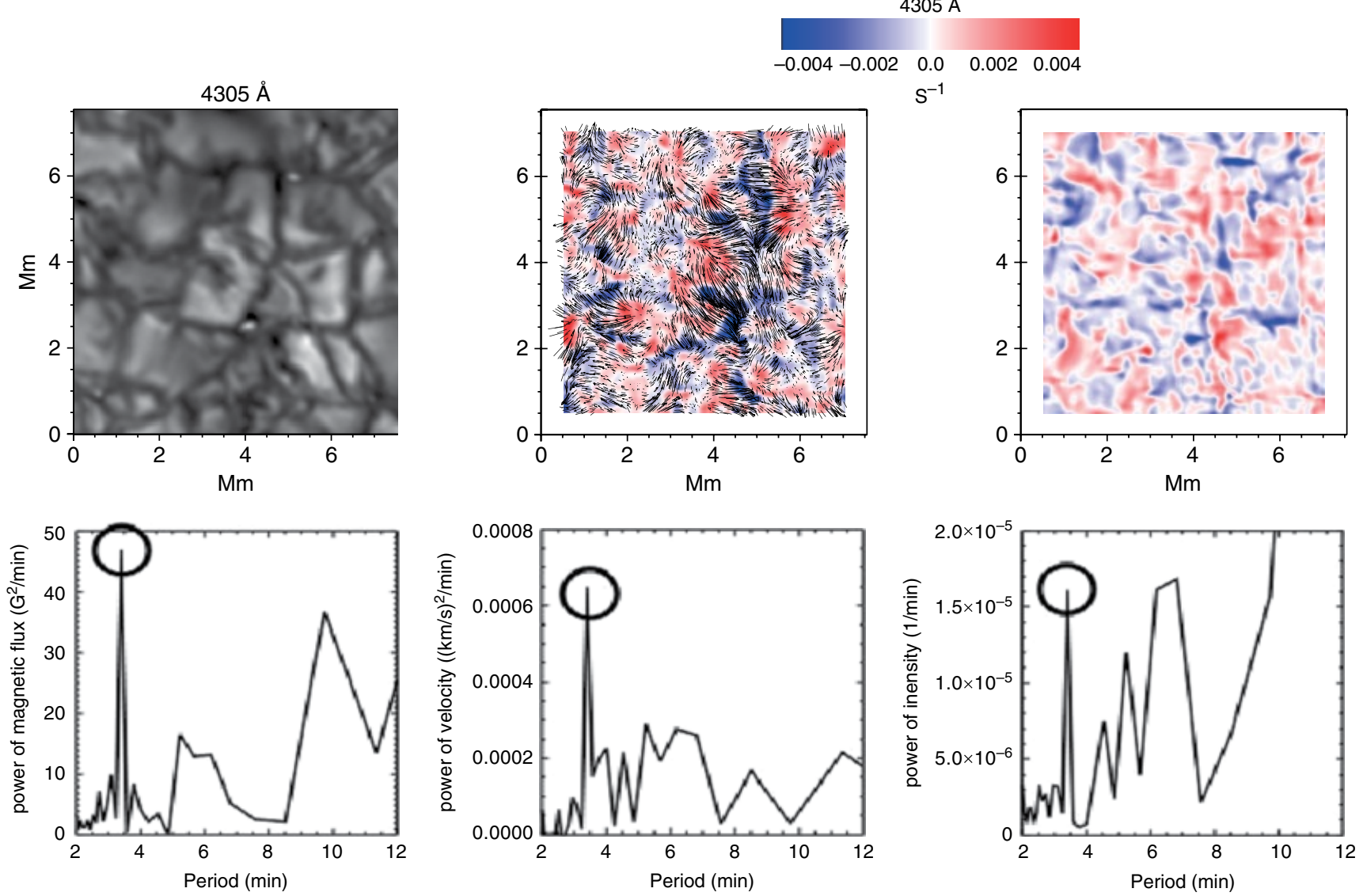

Figure 26.5 Small sub-field of a ROSA G-band image (upper left), obtained using the DST on 29 September 2010. MBPs can be seen as intensity enhancements within the intergranular lanes. The uppermiddle panel displays the simultaneous velocity vectors and divergence of photospheric flows. The arrows indicate the averaged velocity vectors determined from the local correlation tracking of G-band image sequences. The upperright panel displays vorticity $\left(s^{-1}\right.$, where, e.g., $0.002 \mathrm{~s}^{-1}$ represents $\approx 0.11^{\circ} \mathrm{s}^{-1}$ in the clockwise direction) calculated from the derived horizontal photospheric flows. It is clear that the atmosphere in which MBPs are embedded is replete with significant horizontal flows and torsional motion. The lower panels, from lefttoright, display Fourier power spectra of the line-of-sight magnetic flux, the line-of-sight velocity, and the intensity of small-scale magnetic elements (including MBPs that are prevalent in the upperleft panel) observed by the SOT onboard Hinode. The black circles indicate narrow (common) peaks that represent the observational evidence of magnetoacoustic waves in the lower solar atmosphere, including sausage modes, which highlight the ubiquitous nature of wave phenomena across all observational datasets. Images have been adapted from Morton et al. [2013] and Fujimura and Tsuneta [2009].

the majority of the transverse oscillatory periods were in the range of $1-12 \mathrm{mHz}(85-1000 \mathrm{~s})$, there was no specific features unifying the wave phenomena originating within different magnetic structures. As a result the authors concluded that the spectral characteristics represented a unique signature of each magnetic element itself rather than an overarching relationship that defines a collective of small-scale magnetic structures.

Most recently Stangalini et al. [2014] employed a long time series of high-resolution photospheric magnetograms to study the effects of turbulent convection on the excitation of kink oscillations in small-scale magnetic elements. Importantly, the authors utilized empirical mode decomposition techniques, which allowed them to more accurately analyze nonstationary time series that may be dominated by the horizontal displacements of magnetic flux tubes, which are continuously advected and dispersed by granular flows. Subharmonics of fundamental kink oscillations, with periodicities of $7.6 \pm 0.2 \mathrm{~min}$, were used to verify the hypothesis that kink waves are induced through the buffeting of magnetic field lines lying at the boarders of photospheric convective cells. An important aspect of this work is the potential for such kink oscillations to be excited via nonlinear interactions. The presence of period-doubling cascades in the observational results can be interpreted as a signature of chaotic 
excitations in nonlinear systems [e.g., Sander and Yorke, 2009, 2010]. This has important implications for the generation of a wide spectrum of viable propagating periodicities in the lower solar atmosphere, particularly if the driving (i.e., buffeting) frequencies are at values that are not necessarily multiples or fractions of the flux tube's natural frequency. Therefore the work of Stangalini et al. [2014] tentatively suggests that a broad spectrum of wave periodicities in small-scale magnetic elements can be generated regardless of the input motions.

\subsection{ALFVÉN WAVES}

Modern MHD simulations of the lower solar atmosphere clearly show how torsional motions can easily be induced in magnetic elements in the photosphere through the processes of vortical motions and/or buffeting by neighboring granules [Matsumoto and Shibata, 2010; Fedun et al., 2011b; Vigeesh et al., 2012]. The theoretically driven work of van Ballegooijen et al. [2011] and AsgariTarghi and van Ballegooijen [2012] suggested that random displacements of the photospheric anchor points of the magnetic field lines, with velocities on the order of $1.5 \mathrm{kms}^{-1}$, would be sufficient to induce significant wave turbulence, thus potentially creating an efficient dissipation mechanism for Alfvén waves. Chitta et al. [2012b] provided indirect evidence of this effect by comparing the velocity correlation functions of small-scale magnetic elements in the photosphere. The authors detected significant power associated with high-frequency $(>0.02 \mathrm{~Hz} ;<50 \mathrm{~s})$ horizontal motions, and suggested that these cases may be especially important in the creation of a turbulent environment that efficiently promotes Alfvén wave dissipation.

Aside from the numerous theoretical studies related to Alfvén wave generation and dissipation, and as documented by Mathioudakis et al. [2013], observationally identifying pure torsional Alfvén waves in the solar atmosphere has been a monumental struggle ever since they were postulated by Alfvén [1942]. In their most simplistic form (i.e., the torsional Alfvén wave with azimuthal wave number $m=0$ ), their incompressible nature provides the inability to detect them through typical intensity (i.e., density) measurements, and the azimuthal nature of the oscillation provides no transversal deflection of the magnetic structure about its central axis. Furthermore the narrow nature of magnetic flux tubes in the lower solar atmosphere causes difficulties when attempting to resolve the intrinsic blue- and red-shifts associated with velocity measurements at opposite edges of the structure. However, the torsional motion of a magnetic element carrying an Alfvén wave will induce a degree of nonthermal line broadening when observed using spectroscopic techniques [Zaqarashvili, 2003]. The magnitude of the broadening will depend on the velocity amplitude of the torsional mode, which may also be compounded by bulk flows and/or turbulence embedded in the plasma.

Employing the high-resolution spectral imaging capabilities of the Solar Optical Universal Polarimeter (SOUP) [Title et al., 1986] on the SST, Jess et al. [2009] were able to identify periodic nonthermal line broadening associated with a torsional Alfvén wave embodied in the magnetic field lines anchored into a conglomeration of photospheric MBPs (Figure 26.6). Importantly, their interpretation was further strengthened by the fact that the magnetic element did not display any periodic fluctuations in intensity or longitudinal/transverse velocity, which helped verify the absence of other magnetoacoustic modes. In follow-up work, Mathioudakis et al. [2013] revealed the independent and opposite Doppler shifts associated with the opposing edges of the magnetic element, thus reinforcing the interpretation that the observational signatures represented a torsional Alfvén wave. In this work, it was found that the amplitude of the nonthermal broadening was $\sim 0.05 \AA$, equating to a velocity amplitude of $\sim 2.5 \mathrm{kms}^{-1}$. When combined with a local Alfvén speed on the order of $22 \mathrm{kms}^{-1}$ and a plasma density of approximately $1 \times 10^{-9} \mathrm{gcm}^{-3}$, the resulting wave energy was estimated as $\sim 150000 \mathrm{Wm}^{-2}$. While this is a vast quantity of available energy, far in excess of the threshold required to sustain localised chromospheric and coronal heating, the true importance can only be ascertained once the filling factor of such waves is accurately known (e.g., see Chapter 25). However, as documented by Goossens et al. [2011] and Mathioudakis et al. [2013], Alfvén waves are naturally difficult to dissipate unless they are able to find an alternative mechanism to promote efficient energy dissipation. Such mechanisms include phase mixing and resonant absorption [e.g., Goossens and De Groof, 2001], whereby non-uniformities in the magnetic field configurations results in the coupling between neighboring magnetic iso-surfaces, thus promoting a significantly steep gradient to allow efficient energy dissipation. Other possibilities are the mode conversion of Alfvén waves into magnetoacoustic modes that can propagate obliquely to the magnetic field lines, thus promoting efficient energy loss [e.g., Parker, 1991; Nakariakov et al., 1997], or turbulent mixing as a result of highfrequency fluctuations [e.g., van Ballegooijen et al., 2011; Asgari-Targhi and Van Ballegooijen, 2012].

Utilizing the same dataset as presented by Jess et al. [2009], Fedun et al. [2011b] examined the spatial structuring of the observed torsional Alfvén frequencies and related these to the outputs of nonlinear 3D magnetohydrodynamic numerical simulations from the Sheffield Advanced Code (SAC) [Shelyag et al., 2008]. The authors implemented a vortex driver at the base of the simulated domain (see the left-hand image of Figure 26.6) and 

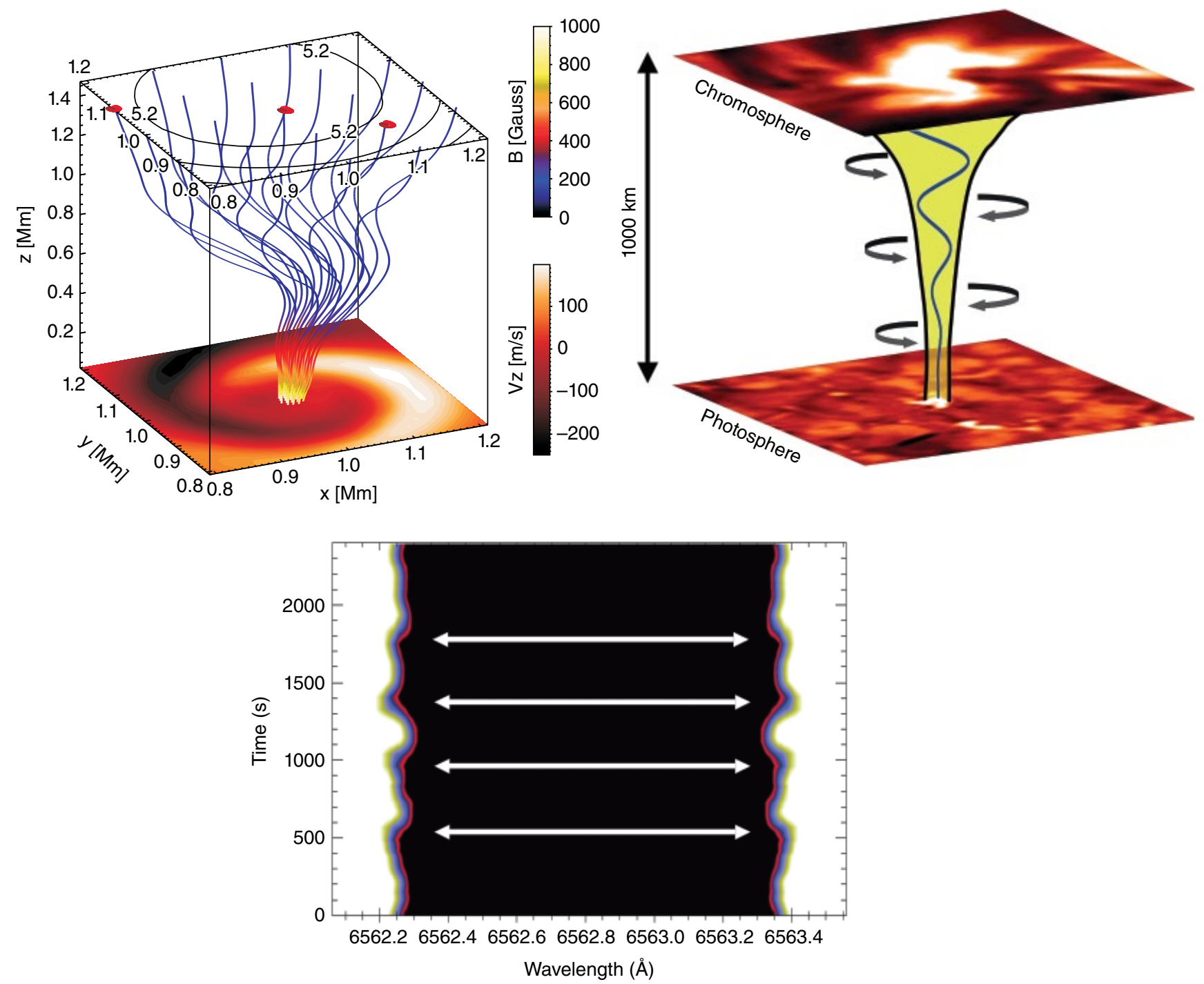

Figure 26.6 The upper left panel displays a 3D snapshot of MHD wave propagation in an open magnetic flux tube. The simulated image is obtained using the nonlinear magnetohydrodynamic SAC code, where the thin multicolor curves represent the magnetic field lines that are scaled using typical field strengths synonymous with MBPs (i.e., $0-1000 \mathrm{G})$. The upper and lower color bars correspond to the magnitude of the magnetic field and the vertical velocity, $V_{z^{\prime}}$ at the level of the photospheric driver, resepctively. Iso-contours of the magnetic field are displayed as solid black lines which are labeled in the top horizontal slice taken at an atmospheric height of $1.4 \mathrm{Mm}$ (or $1400 \mathrm{~km}$ ). The bottom of the 3D grid displays a horizontal cross-cut through the location of the torsional driver. The upper right panel displays a typical expanding magnetic flux tube sandwiched between photospheric (broadband $\mathrm{H} \alpha$ ) and chromospheric (narrowband $\mathrm{H} \alpha$ ) intensity images obtained with the SST on 23 August 2007. The observational dataset revealed that the magnetic flux tube underwent a torsional Alfvénic perturbation, indicated by the periodic nonthermal spectral broadening displayed in the lower panel. In the wavelength-time plot the $\mathrm{H} \alpha$ absorption profile line width, calculated using spectral imaging techniques, is observed to oscillate with a periodicity $\sim 420$ s, with consecutive peaks indicated by the white arrows. As can be seen in both upper panels, the Alfvénic displacements are torsional motions that remain perpendicular to both the direction of propagation and the magnetic fields outlining constant magnetic surfaces. Images adapted from Jess et al. [2009] and Fedun et al. [2011b].

revealed how magnetic flux tubes can act as a spatial frequency filter for torsional Alfvén waves. Importantly, the authors found that his form of frequency filtering is strongly dependent on the structure and geometry of the magnetic field itself. This implies that the observed spatial wave power and oscillatory frequencies can be a function of the underlying MBP, possibly allowing magnetic fields to be mapped as a function of atmospheric 
height solely employing such seismology techniques [Fedun et al., 2011b].

Even with higher sensitivity equipment now becoming commonplace on a majority of ground-based telescopes (e.g., the IBIS and CRISP imaging spectropolarimeters; Cavallini [2006]; Scharmer et al. [2008]), there is still a significant lack of subsequent Alfvén wave detections in the lower solar atmosphere. This is in stark contrast to modern numerical simulations (e.g., the MuRAM code; Vögler et al. [2005]), which indicate widespread torsional motions in synthesized photospheric filtergrams [Shelyag et al., 2013]. Instead, many have turned their attention to the chromosphere where the interaction, mode conversion, and dissipation of Alfvén waves might have more identifiable signatures, particularly in regions where the plasma $\beta$ changes abruptly, or self-induced turbulence results in rapid localised dissipation [van Ballegooijen et al., 2011; Asgari-Targhi and van Ballegooijen, 2012].

\subsection{CONCLUSIONS AND FUTURE DIRECTIONS}

In the near future it is expected that high sensitivity 2D spectropolarimeters (e.g., CRISP and IBIS) will be employed simultaneously alongside high-cadence imagers (e.g., Hinode/SOT and ROSA) to obtain multiwavelength time series at the highest spatial, temporal and spectral resolutions currently achievable (e.g., see Figure 26.7).
High precision measurements will allow the characterization of MHD waves themselves manifesting in small-scale magnetic elements that are at the limits of current telescope resolving power. Important oscillatory parameters, such as the propagation speeds, amplitudes, and phase relationships will allow MHD wave phenomena to be documented with unprecedented accuracy, including the establishment of evidence to verify the presence of standing/propagating, fast/slow, trapped/ leaky, and surface/body oscillatory modes. Furthermore the multiwavelength nature of the data will also enable the detected MHD waves to be tracked through the solar atmosphere as they journey from the photosphere, through the tenuous chromosphere, and into the superheated corona. Importantly, coverage of the waves as they propagate through the different atmospheric regions where the plasma $\beta$ changes from magnetically dominated to plasma pressurized regimes will provide valuable insight on aspects of mode coupling and wave dissipation. Many previous examples have indicated that regions where $\beta=1$, often in locations sandwiched between the photosphere and chromosphere, provides opportune atmospheric conditions to promote efficient oscillatory mode conversion, thus allowing naturally difficult-todissipate waves (e.g., Alfvén waves) to convert into more readily dissipated compressible modes [e.g., Ulmschneider et al., 1991; Kalkofen, 1997; Hasan et al., 2003; McAteer
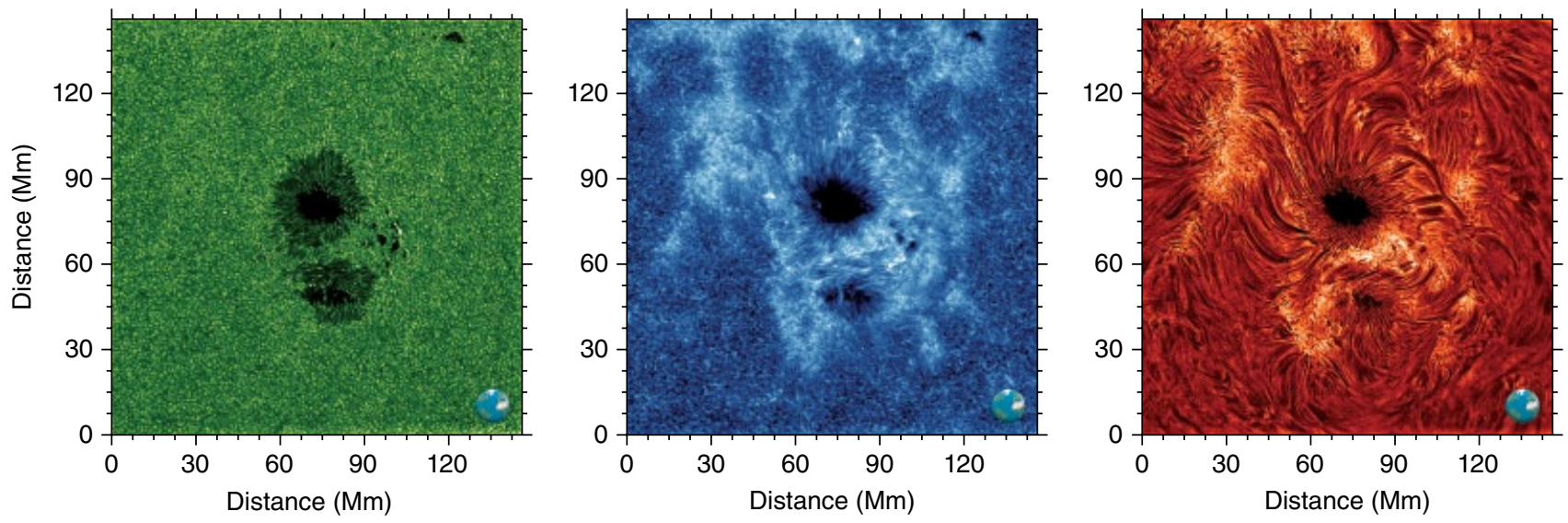

Figure 26.7 Simultaneous images of a solar active region, captured using both existing and next-generation imaging detectors on the DST during 24 August 2014. The left and middle images are G-band (photosphere) and Ca II K (upper photosphere/lower chromosphere) snapshots, respectively, acquired using the electron-multiplying CCD cameras of the ROSA multi-camera system. A true-size representation of the Earth is depicted in the lower right section of each image to provide a sense of scale. The image on the right reveals the solar chromosphere, captured through a 0.25 $\mathrm{H} \alpha$ core filter, and employs a 4.2 MP Zyla CMOS sensor from Andor Technology, which allows image sequences to be obtained with $15 \mathrm{~ms}$ exposure times and frame rates exceeding $60 \mathrm{~s}^{-1}$. Furthermore the large pixel formats of modern CCD and CMOS detectors allow fields of view in excess of $200^{\prime \prime} \times 200^{\prime \prime}$ to be sampled at the diffraction limit, and when combined with numerous detectors each sampling discreet wavelengths, provides a seamless view through the tenuous atmospheric layers. Combining such high-sensitivity detectors with current and future telescope facilities will open the door for greater scientific understanding through drastically improved number statistics and larger fields of view. Images courtesy of D. B. Jess. 
et al., 2003; Bloomfield et al., 2006; Goossens et al., 2006; Jess et al., 2012b].

In addition to maximizing the scientific return of the current fleet of ground- and space-based solar telescopes, the solar community eagerly awaits the arrival of the first next-generation, high-resolution facilities, including the $2 \mathrm{~m}$ National Large Solar Telescope (NLST) [Hasan et al., 2010] in Ladakh, India, and the $4 \mathrm{~m}$ Daniel $\mathrm{K}$. Inouye Solar Telescope (DKIST, formerly the Advanced Technology Solar Telescope, ATST) [Keil et al., 2003; Rimmele et al., 2010] atop the Haleakalā volcano on the Pacific island of Maui. These pioneering facilities are due to receive first light toward the latter stages of this decade, and by utilizing dramatically increased aperture sizes ( $4 \mathrm{~m}$ in the case of DKIST), photospheric structures down to $\sim 20 \mathrm{~km}$ in size will be able to be detected, tracked and studied in unprecedented detail. In the longer term is the launch of the Solar-C payload (expected launch date around 2019) with a UV/visible/IR telescope approaching $1.4 \mathrm{~m}$ in diameter [Suematsu et al., 2014]. The seeing free data from a high-resolution space-based facility will enable near-continuous observations of magnetic elements to be obtained without the need for extensive postprocessing algorithms. Even more long-term is the $4 \mathrm{~m}$ European Solar Telescope (EST) [Collados et al., 2010] and the 5-8 m Chinese Giant Solar Telescope (CGST) [Liu et al., 2014], which are expected to begin construction following 2020, and allow structures as small as $\sim 10 \mathrm{~km}$ in size to be observed for the first time.

The desire to improve not only the spatial resolution of solar telescopes, but also the temporal and spectral resolutions through the development of new imaging and spectropolarimetric instruments, will enable key outstanding questions related to lower atmospheric understanding to be firmly addressed. Such questions include:

- Do all small-scale magnetic elements carry energy in the form of MHD waves? There is an abundance of evidence that demonstrates the suitability of narrow magnetic strands as efficient wave/energy conduits. For example, Jess et al. [2012c] found that $73 \%$ of MBP structures demonstrated magnetoacoustic wave signatures. However, does this mean that $\sim 27 \%$ of these features contain no oscillatory phenomena? Or do waves exist, but under different guises (e.g., the more difficult to detect Alfvén modes)?

- What is the dominant mode of oscillation present in small-scale photospheric magnetic features? The majority of research to date has focussed on the magnetoacoustic signatures detectable through intensity (i.e., density) fluctuations. However, with improved observations and detection algorithms [e.g., Stangalini et al., 2013a, b], it has become clear that other wave modes may be present in small-scale magnetic elements alone or alongside their compressible counterparts. Are these additional modes (e.g., kink and Alfvén waves) superimposed on top of the seemingly ubiquitous compressive oscillations? Or are they the dominant mode of oscillation in certain magnetic features? And if so, which features?

- How do the $\beta=1$ layers contribute to both the waveforms visible in the outer solar atmosphere and the rate of localized heating through wave dissipation? It is well accepted that regions of the Sun's atmosphere where $\beta=1$ (i.e., the magnetic pressure is equal to the gas pressure) are optimal for oscillatory mode conversion [e.g., Ulmschneider et al., 1991; Kalkofen, 1997; Hasan et al., 2003]. McAteer et al. [2003] and Bloomfield et al. [2006] have demonstrated the coupling between kink waves and longitudinal oscillations, while Jess et al. [2012b] have documented the conversion between longitudinal waves and kink/sausage modes. Regions where $\beta=1$ are also predicted to have important consequences for Alfvén wave conversion through processes such as resonant absorption [e.g., Goossens et al., 2006]. Can such atmospheric layers convert observed (and possibly unobserved!) waves into other modes that can readily dissipate their energy into the surrounding plasma? Do the preferred conversion mechanisms substantiate the abundance of oscillatory motion observed in the outer regions of the solar atmosphere?

- Can we find evidence for the existance of magnetoacoustic wave modes with azimuthal wave numbers exceeding 1 ? The observational work to date suggests an abundance of sausage $(m=0)$ and kink $(m=1)$ mode oscillations in the lower solar atmosphere. However, theoretical predictions also suggest that higher order wave numbers (i.e., fluting modes with $m \geq 2$ ) should also be prevalent in magnetic flux tubes (for a recent review, see Goossens et al. [2011], and the references therein). Why do we currently have no evidence for such oscillations? Is it a result of relatively poor telescope resolution and/or instrument sensitivity? Or are the amplitudes of these oscillatory modes so small that they become impossible to disentangle from other superimposed waves?

- What role do downwardly propagating MHD waves play in the structuring, evolution, dynamics, and energy balance of magnetic features in the solar photosphere? Attempting to find the elusive solution to the coronal heating problem has resulted in an overwhelming interest in upwardly propagating MHD waves. However, observations continue to identify significant oscillations propagating downward through the solar atmosphere [e.g., Gupta et al., 2013]. Jess et al. [2012c] estimated that approximately a quarter of all detected magnetoacoustic waves in MBPs were downwardly propagating. Are these propagating oscillations generated at higher atmospheric heights? Are they the counterpart of upwardly propagating oscillations generated at the opposite foot point of a 
closed loop? Or are they the result of reflection as upwardly propagating waves encounter the severe density discontinuities intrinsic to chromospheric and transition region layers? Furthermore, how do these downward motions contribute to the mass/energy flow associated with magnetic elements in general?

The questions above are not intended to highlight all of the outstanding problems related to MHD wave phenomena in the lower solar atmosphere, nor are they listed in order of importance. Instead, they are simply listed as those that we feel have an overarching central importance when attempting to address the long-standing issues of wave generation, propagation, energy transfer, and dissipation throughout the photosphere and beyond. Furthermore it seems unlikely that the questions above can be unequivocally answered relying solely on observational approaches. A combination of theoretical, analytical, and numerical modeling techniques will be required to help extract and interpret the wealth of MHD wave modes that exist in the lower solar atmosphere. Thus the answers to the key science questions outlined above will only arise through the novel use of high-resolution (spatial, temporal, and spectral) photospheric datasets alongside the rapid development of our theoretical understanding.

\section{ACKNOWLEDGMENTS}

D. B. J. wishes to thank the UK Science and Technology Facilities Council (STFC) for the award of an Ernest Rutherford Fellowship alongside a dedicated Research Grant. G. V. acknowledges the support of the Leverhulme Trust (UK).

\section{REFERENCES}

Alfvén, H. (1942), Nature, 150, 405.

Andić, A. (2007), Sol. Phys., 243, 131.

Andić, A., Chae, J., Park, H., et al. (2013), Sol. Phys., 288, 55.

Arber, T. D., Longbottom, A. W., Gerrard, C. L., and Milne, A. M. (2001), J. Comput. Phys., 171, 151.

Asgari-Targhi, M., and van Ballegooijen, A. A. (2012), Astrophys. J., 746, 81.

Bel, N., and Leroy, B. (1977), Astron. Astrophys., 55, 239.

Bharti, L., Cameron, R. H., Rempel, M., Hirzberger, J., and Solanki, S. K. (2012), Astrophys. J., 752, 128.

Bloomfield, D. S., McAteer, R. T. J., Mathioudakis, M., and Keenan, F. P. (2006), Astrophys. J.,652, 812.

Carlsson, M., Hansteen, V. H., de Pontieu, B., et al. (2007), PASJ, 59, 663.

Cauzzi, G., Falchi, A., and Falciani, R. (2000), Astron. Astrophys, 357,1093

Cavallini, F. (2006), Sol. Phys., 236, 415.

Chitta, L. P., Jain, R., Kariyappa, R., and Jefferies, S. M. (2012a), Astrophys. J., 744, 98.
Chitta, L. P., van Ballegooijen, A. A., Rouppe van der Voort, L., DeLuca, E. E., and Kariyappa, R. (2012b), Astrophys. J., 752,48

Chou, D.-Y., Liang, Z.-C., Yang, M.-H., Zhao, H., and Sun, M.-T. (2009), Astrophys. J., 696, L106.

Choudhuri, A. R., Auffret, H., and Priest, E. R. (1993a), Sol. Phys., 143, 49.

Choudhuri, A. R., Dikpati, M., and Banerjee, D. (1993), Astrophys. J., 413, 811.

Collados, M., Bettonvil, F., Cavaller, L., et al. (2010), Astron. Nachrichten, 331, 615.

Couvidat, S. (2013), Sol. Phys., 282, 15.

Cranmer, S. R. and van Ballegooijen, A. A. (2005), Astrophys. J. (Supp.), 156, 265.

Crockett, P. J., Mathioudakis, M., Jess, D. B., et al. (2010), Astrophys. J., 722, L188.

De Moortel, I., and Hood, A. W. (2003), Astron. Astrophys, 408, 755 .

De Moortel, I., and Hood, A. W. (2004), Astron. Astrophys., 415,705 .

De Pontieu, B., Erdélyi, R., and James, S. P. (2004), Nature, 430, 536.

de Wijn, A. G., McIntosh, S. W., and De Pontieu, B. (2009), Astrophys. J., 702, L168.

Deforest, C. E., and Gurman, J. B. (1998), Astrophys. J., 501, L217.

Deubner, F.-L. (1971), Sol. Phys., 17, 6.

Deubner, F.-L., and Liedler, R. (1969), Sol. Phys., 7, 87.

Dunn, R. B., and Zirker, J. B. (1973), Sol. Phys., 33, 281.

Duvall, T. L., Jr., Jefferies, S. M., Harvey, J. W., and Pomerantz, M. A. (1993), Nature, 362, 430.

Edwin, P. M., and Roberts, B. (1983), Sol. Phys., 88, 179.

Fedun, V., Shelyag, S., and Erdélyi, R. (2011a), Astrophys. J., $727,17$.

Fedun, V., Verth, G., Jess, D. B., and Erdélyi, R. (2011b), Astrophys. J., 740, L46.

Fujimura, D., and Tsuneta, S. (2009), Astrophys. J., 702, 1443.

Goossens, M., Andries, J., and Arregui, I. (2006), Roy. Soc. London Phil. Trans. Series A, 364, 433.

Goossens, M., and De Groof, A. (2001), Phys. Plasmas, 8, 2371.

Goossens, M., Erdélyi, R., and Ruderman, M. S. (2011), Space Sci. Rev., 158, 289.

Goossens, M., Terradas, J., Andries, J., Arregui, I., and Ballester, J. L. (2009), Astron. Astrophys., 503, 213.

Gupta, G. R., Subramanian, S., Banerjee, D., Madjarska, M. S., and Doyle, J. G. (2013), Sol. Phys., 282, 67.

Handy, B. N., Acton, L. W., Kankelborg, C. C., et al. (1999), Sol. Phys., 187, 229.

Hansteen, V. H., De Pontieu, B., Rouppe van der Voort, L., van Noort, M., and Carlsson, M. (2006), Astrophys. J., 647, L73.

Hasan, S. S., Kalkofen, W., van Ballegooijen, A. A., and Ulmschneider, P. (2003), Astrophys. J., 585, 1138.

Hasan, S. S., Soltau, D., Kärcher, H., Süß, M., and Berkefeld, T. (2010), Astron. Nachrichten, 331, 628.

Hewitt, R. L., Shelyag, S., Mathioudakis, M., and Keenan, F. P. (2014), Astron Astrophys., 565, A84.

Ilonidis, S., and Zhao, J. (2011), Sol. Phys., 268, 377.

Isobe, H., and Tripathi, D. (2006), Astron Astrophys., 449, L17.

Isobe, H., Tripathi, D., Asai, A., and Jain, R. (2007), Sol. Phys., 246,89 . 
Jackiewicz, J., and Balasubramaniam, K. S. (2013), Astrophys. $J ., 765,15$.

Jess, D. B., Andić, A., Mathioudakis, M., Bloomfield, D. S., and Keenan, F. P. (2007), Astron. Astrophys., 473, 943.

Jess, D. B., De Moortel, I., Mathioudakis, M., et al. (2012a), Astrophys. J., 757, 160.

Jess, D. B., Mathioudakis, M., Browning, P. K., Crockett, P. J., and Keenan, F. P. (2010a), Astrophys. J., 712, L111.

Jess, D. B., Mathioudakis, M., Christian, D. J., Crockett, P. J., and Keenan, F. P. (2010b), Astrophys. J., 719, L134.

Jess, D. B., Mathioudakis, M., Christian, D. J., et al. (2010c), Sol. Phys., 261, 363.

Jess, D. B., Mathioudakis, M., Erdélyi, R., et al. (2009), Science, $323,1582$.

Jess, D. B., Pascoe, D. J., Christian, D. J., et al. (2012b), Astrophys. J., 744, L5.

Jess, D. B., Shelyag, S., Mathioudakis, M., et al. (2012c), Astrophys. J., 746, 183.

Kalkofen, W. (1997), Astrophys. J., 486, L145.

Kalkofen, W. (2007), Astrophys. J., 671, 2154.

Kalkofen, W. (2008), J. Astrophys. Astron., 29, 163.

Kato, Y., Steiner, O., Steffen, M., and Suematsu, Y. (2011), Astrophys. J., 730, LL24.

Keil, S. L., Rimmele, T., Keller, C. U., et al. (2003), Proc. SPIE, 4853, 240

Keys, P. H., Mathioudakis, M., Jess, D. B., et al. (2011), Astrophys. J., 740, L40.

Khomenko, E., Collados, M., and Felipe, T. (2008), Sol. Phys., 251, 589.

Khomenko, E. V., Collados, M., Solanki, S. K., Lagg, A., and Trujillo Bueno, J. (2003), Astron. Astrophys., 408, 1115.

Kim, Y.-H., Bong, S.-C., Park, Y.-D., et al. (2008), J. Korean Astron. Soc., 41, 173.

Kostik, R., and Khomenko, E. (2013), Astron. Astrophys., 559, A107.

Kosugi, T., Matsuzaki, K., Sakao, T., et al. (2007), Sol. Phys., $243,3$.

Kuridze, D., Verth, G., Mathioudakis, M., et al. (2013), Astrophys. J., 779, 82.

Lawrence, J. K., and Cadavid, A. C. (2010), Sol. Phys., 261, 35.

Lawrence, J. K., and Cadavid, A. C. (2012), Sol. Phys., 280, 125.

Lawrence, J. K., Cadavid, A. C., Christian, D. J., Jess, D. B., and Mathioudakis, M. (2011), Astrophys. J., 743, L24.

Leighton, R. B. (1960), IAUS, 12, 321.

Leighton, R. B., Noyes, R. W., and Simon, G. W. (1962), Astrophys. J., 135, 474.

Li, T., and Zhang, J. (2012), Astrophys. J., 760, L10.

Lin, H. (1995), Astrophys. J., 446, 421.

Liu, Z., Jin, Z., Yuan, S., et al. (2014), Proc. SPIE, 9145, 914526.

Lopes, I., and Silk, J. (2014), Astrophys J., 794, 32.

McAteer, R. T. J., Gallagher, P. T., Williams, D. R., et al. (2003), Astrophys. J., 587, 806.

Martínez González, M. J., Asensio Ramos, A., Manso Sainz, R., et al. (2011), Astrophys. J., 730, L37.

Martínez González, M. J., Collados, M., Ruiz Cobo, B., and Beck, C. (2008), Astron. Astrophys., 477, 953.

Martínez Pillet, V., Del Toro Iniesta, J. C., Álvarez-Herrero, A., et al. (2011), Sol. Phys., 268, 57.
Mathioudakis, M., Jess, D. B., and Erdélyi, R. (2013), Space Sci. Rev., 175, 1.

Matsumoto, T., and Shibata, K. (2010), Astrophys. J., 710, 1857.

Mein, N., and Mein, P. (1976), Sol. Phys., 49, 231.

Moreels, M. G., and Van Doorsselaere, T. (2013a), Astron. Astrophys., 551, A137.

Moreels, M. G., Goossens, M., and Van Doorsselaere, T. (2013b), Astron. Astrophys., 555, A75.

Morton, R. J., Erdélyi, R., Jess, D. B., and Mathioudakis, M. (2011), Astrophys. J., 729, L18.

Morton, R. J., Verth, G., Fedun, V., Shelyag, S., and Erdélyi, R. (2013), Astrophys. J., 768, 17.

Morton, R. J., Verth, G., Hillier, A., and Erdélyi, R. (2014), Astrophys. J., 784, 29.

Morton, R. J., Verth, G., Jess, D. B., et al. (2012), Nature Comm., 3.

Musielak, Z. E., and Ulmschneider, P. (2003), Astron. Astrophys., 406, 725.

Nagashima, K., Sekii, T., Kosovichev, A. G., et al. (2007), PASJ, 59,631 .

Nakariakov, V. M., Roberts, B., and Murawski, K. (1997), Sol. Phys., 175, 93.

Narayan, G. (2011), Astron. Astrophys., 529, AA79.

Noyes, R. W., and Leighton, R. B. (1963), Astrophys. J., 138, 631.

Ofman, L., Nakariakov, V. M., and Deforest, C. E. (1999), Astrophys. J., 514, 441.

Ofman, L., and Wang, T. (2002), Astrophys. J., 580, L85.

Osterbrock, D. E. (1961), Astrophys. J., 134, 347.

Parchevsky, K. V., and Kosovichev, A. G. (2007), Astrophys. J., 666, L53.

Parker, E. N. (1991), Astrophys. J., 376, 355.

Rimmele, T. R., and Marino, J. (2011), Living Rev. Solar Phys., $8,2$.

Rimmele, T. R., Wagner, J., Keil, S., et al. (2010), Proc. SPIE, 7733.

Roberts, B. (1983), Sol. Phys., 87, 77.

Ryutova, M., Berger, T, and Title, A. (2008), Astrophys. J., 676, 1356.

Sánchez Almeida, J., Bonet, J. A., Viticchié, B., and Del Moro, D. (2010), Astrophys. J., 715, L26.

Sander, E., and Yorke, J. A. (2009), arXiv:0910.3570.

Sander, E., and Yorke, J. A. (2010), arXiv:1002.3363.

Scharmer, G. B., Narayan, G., Hillberg, T., et al. (2008), Astrophys. J., 689, L69.

Schou, J., Antia, H. M., Basu, S., et al. (1998), Astrophys. J., $505,390$.

Shelyag, S., Cally, P. S., Reid, A., and Mathioudakis, M. (2013), Astrophys. J., 776, LL4.

Shelyag, S., Fedun, V., and Erdélyi, R. (2008), Astron. Astrophys., 486, 655 .

Shen, Y., Ichimoto, K., Ishii, T. T., et al. (2014), Astrophys. J., $786,151$.

Solanki, S. K. (1993), Space Sci. Rev., 63, 1.

Spruit, H. C. (1976), Sol. Phys., 50, 269.

Stangalini, M., Berrilli, F., and Consolini, G. (2013a), Astron. Astrophys., 559, A88.

Stangalini, M., Consolini, G., Berrilli, F., De Michelis, P., and Tozzi, R. (2014), Astron. Astrophys., 569, A102. 
Stangalini, M., Del Moro, D., Berrilli, F., and Jefferies, S. M. (2011), Astron. Astrophys., 534, A65.

Stangalini, M., Solanki, S. K., Cameron, R., and Martínez Pillet, V. (2013b), Astron. Astrophys., 554, A115.

Steiner, O., Grossmann-Doerth, U., Knölker, M., and Schüssler, M. (1998), Astrophys. J., 495, 468.

Stenflo, J. O. (1985), Sol. Phys., 100, 189.

Suematsu, Y., Katsukawa, Y., Hara, H., et al. (2014), Proc. SPIE, 9143, 91431P.

Suematsu, Y., Tsuneta, S., Ichimoto, K., et al. (2008), Sol. Phys., 249, 197.

Suzuki, T. K. and Inutsuka, S.-I. (2005), Astrophys. J., 632, L49.

Thomas, J. H., Cram, L. E., and Nye, A. H. (1982), Nature, 297, 485.

Title, A. M., Tarbell, T. D., Acton, L., Duncan, D., and Simon, G. W. (1986), Adv. Space Res., 6, 253.

Title, A. M., Topka, K. P., Tarbell, T. D., et al. (1992), Astrophys. J., 393, 782.

Tomczyk, S., and McIntosh, S. W. (2009), Astrophys. J., 697, 1384.

Tritschler, A., Schmidt, W., Langhans, K., and Kentischer, T. (2002), Sol. Phys., 211, 17.

Tsuneta, S., Ichimoto, K., Katsukawa, Y., et al. (2008), Sol. Phys., 249, 167.
Ulmschneider, P. (1976), Sol. Phys., 49, 249.

Ulmschneider, P., Zaehringer, K., and Musielak, Z. E. (1991), Astron. Astrophys., 241, 625.

Ulrich, R. K. (1970), Astrophys. J., 162, 993.

Utz, D., Jurčák, J., Hanslmeier, A., et al. (2013), Astron. Astrophys., 554, A65.

van Ballegooijen, A. A., Asgari-Targhi, M., Cranmer, S. R., and DeLuca, E. E. (2011), Astrophys. J., 736, 3.

van Noort, M., Rouppe van der Voort, L., and Löfdahl, M. G. (2005), Sol. Phys., 228, 191.

Verdini, A., and Velli, M. (2007), Astrophys. J., 662, 669.

Verth, G., Goossens, M., and He, J.-S. (2011), Astrophys. J., 733, L15.

Vigeesh, G., Fedun, V., Hasan, S. S., and Erdélyi, R. (2012), Astrophys. J., 755, 18.

Vögler, A., Shelyag, S., Schüssler, M., et al. (2005), Astron. Astrophys., 429, 335.

Wedemeyer-Böhm, S., Steiner, O., Bruls, J., and Rammacher, W. (2007), Phys. Chromosph. Plasmas, 368, 93.

Wöger, F., von der Lühe, O., and Reardon, K. (2008), Astron. Astrophys., 488, 375.

Woods, D. T., and Cram, L. E. (1981), Sol. Phys., 69, 233.

Zaqarashvili, T. V. (2003), Astron. Astrophys., 399, L15. 